Meta

Journal des traducteurs

Translators' Journal

\title{
Les termes techniques en interprétation simultanée
}

\section{Daniel Gile}

Volume 30, numéro 3, septembre 1985

URI : https://id.erudit.org/iderudit/002891ar

DOI : https://doi.org/10.7202/002891ar

Aller au sommaire du numéro

Éditeur(s)

Les Presses de l'Université de Montréal

ISSN

0026-0452 (imprimé)

1492-1421 (numérique)

Découvrir la revue

Citer cet article

Gile, D. (1985). Les termes techniques en interprétation simultanée. Meta, 30(3), 199-210. https://doi.org/10.7202/002891ar
Résumé de l'article

L'article passe en revue certaines caractéristiques du vocabulaire spécialisé des conférences techniques et explique leurs incidences sur l'interprétation. Il évoque les différentes tactiques utilisées en cabine pour surmonter les problèmes ainsi que les principaux paramètres affectant la qualité de la prestation de l'interprète en matière terminologique. d'utilisation que vous pouvez consulter en ligne.

https://apropos.erudit.org/fr/usagers/politique-dutilisation/ 


\section{LES TERMES TECHNIQUES EN INTERPRÉTATION SIMULTANÉE}

DANIEL GILE

Résumé : L'article passe en revue certaines caractéristiques du vocabulaire spécialisé des conférences techniques et explique leurs incidences sur l'interprétation. Il évoque les différentes tactiques utilisées en cabine pour surmonter les problèmes ainsi que les principaux paramètres affectant la qualité de la prestation de l'interprète en matière terminologique.

\section{INTRODUCTION}

Les utilisateurs des services d'interprétation ont tendance à considérer que la principale difficulté des conférences techniques est lexicologique, et interrogent souvent les interprètes sur leurs méthodes de travail dans ce domaine.

Les interprètes, quant à eux, s'expliquent peu sur ce point. Dans leurs travaux, ils soulignent surtout la nature intellectuelle de leur démarche, qui est l'exégèse du discours (Seleskovitch 1968, Lederer 1978, Déjean 1978); les aspects techniques de l'interprétation sont laissés de côté.

En fait, il n'est de bonne interprétation technique sans une solide préparation. Le présent article cherche à en expliquer les raisons ; il décrit les différents types d'obstacles terminologiques qui se dressent devant les interprètes, et les tactiques utilisées pour les franchir ou les contourner.

La présente synthèse se fonde sur l'observation sur le terrain et sur des expériences réalisées en laboratoire. L'échantillon étudié se compose de collègues volontaires (pour les expériences) et de collègues ayant travaillé avec l'auteur à des conférences techniques (quelques dizaines au total). Les langues de travail concernées sont également limitées en nombre (français, anglais, allemand, espagnol, japonais, hébreu). Toutefois, la convergence dans les problèmes et démarches, ainsi que les réflexions des collègues ne faisant pas partie de l'échantillon, donnent à penser que l'image dégagée reflète de manière représentative la situation de l'ensemble des interprètes travaillant à des conférences techniques.

\section{LE VOCABULAIRE TECHNIQUE DES CONFÉRENCES}

Le vocabulaire technique dans les conférences internationales a des dimensions très variables : insignifiant dans certaines réunions à caractère politique ou administratif, il atteint couramment plusieurs centaines de mots dans les colloques scientifiques ou techniques.

Par ailleurs, à moins d'avoir une documentation très complète, l'interprète ne peut savoir à l'avance quels seront les mots effectivement employés et doit être prêt à affronter un très vaste vocabulaire potentiel : en effet, quel que soit le thème de la réunion, des mots appartenant à des domaines connexes ou étrangers sont susceptibles d'apparaître. On peut citer à titre d'exemple une récente conférence sur l'innovation technologique dont une session d'une demi-journée consacrée aux applications du laser compor- 
tait des incursions dans la cancérologie, la stomatologie, la gynécologie, les techniques aéronautiques et spatiales, la soudure, les machines-outils...

Aucun interprète ne connaît les dizaines de milliers, voire les centaines de milliers de mots du vocabulaire potentiel d'une conférence - aucun interprète ne peut les apprendre. Or, un mot inconnu ou mal connu peut entraver la compréhension de l'idée, et par là empêcher sa restitution.

Dans les réunions où a lieu un véritable échange, l'interprète peut apprendre le vocabulaire au fil des travaux (Seleskovitch $1968: 155$ ). Par contre, dans les très nombreuses conférences qui se composent d'un enchaînement d'exposés entrecoupés de très brèves discussions, la chose est impossible. Pour cerner et apprendre le vocabulaire, condition indispensable à une bonne qualité du travail, l'interprète dispose d'un seul moyen efficace : la documentation.

Les lettres d'engagement approuvées par l'AIIC (Association internationale des interprètes de conférence) prévoient l'envoi de la documentation nécessaire à la préparation de la réunion à tous les membres de l'équipe.

Quand la documentation est incomplète ou absente, une partie plus ou moins grande du vocabulaire de la conférence reste inconnue de l'interprète, qui s'expose alors à des risques importants de perte informationnelle.

\section{LES PROBLÈMES}

À propos des problèmes susceptibles de se poser durant la première phase de l'interprétation, à savoir l'écoute et l'analyse, il est bon de rappeler le rôle capital des structures probabilistes de réception chez l'auditeur.

Un locuteur ne prononce pas toujours les mots de la même manière, et il n'existe pas d'enchaînement de sons précis qui corresponde universellement à une suite de mots. Dans la conversation, l'auditeur sélectionne certaines caractéristiques du son entendu, les " traits pertinents ", qu'il interprète en fonction de ses attentes (voir Levinson et Liberman 1981).

Du point de vue de la théorie de l'information, la compréhension d'un message implique toujours plus que ce qui est contenu dans le signal lui-même ; elle implique une référence à la totalité des possibilités que le récepteur a à sa disposition et parmi lesquelles le signal en question a été choisi. Cet ensemble de possibilités n'est nullement uniforme, il a un profil caractéristique : certaines possibilités sont plus probables que d'autres, et ces variations dans les degrés de probabilité influencent le processus de décodage du récepteur. Si le récepteur du message sait que les chiffres y sont plus probables que les mots, il pensera que /ka.../ a plus de chances d'être "quatre " que "catastrophes". (Horman 1972 : 78.)

Les termes techniques constituent un signal bref d'une fraction de seconde. Ils sont donc particulièrement vulnérables à la distorsion du son (mauvaise qualité du matériel électronique, fort accent étranger ou régional de l'orateur), au " bruit » (toutes perturbations sonores), et au relâchement de l'attention de l'interprète.

L'importance de ce dernier facteur apparaît clairement à la lumière du concept d'" équilibre d'interprétation ".

En simultanée, l'interprète répartit son énergie entre l'écoute et l'analyse, l'effort de mémoire (certains éléments doivent être retenus pendant un certain temps avant d'être restitués dans la langue d'arrivée), et la production du discours dans la langue d'arrivée. L'équilibre précaire maintenu entre ces trois efforts est facilement rompu : si l'orateur est trop rapide, par exemple, l'interprète prend du retard, ce qui l'oblige à faire un effort de mémoire supplémentaire au détriment de l'écoute, et il n'entend plus très bien la suite du discours (ce concept d'« équilibre d'interprétation » est développé dans 
un autre article à paraître : « Des difficultés de la transmission informationnelle en interprétation simultanée ").

Par ailleurs, si les structures d'attente de l'interprète sont faibles, il risque de ne pouvoir identifier les mots d'après le signal qui lui parvient ; un mot inconnu, mal connu ou inattendu est moins facilement reconstitué qu'un mot familier.

L'identification du mot n'est d'ailleurs qu'un préalable à l'assimilation du sens ; celle-ci n'est possible que si le mot est connu, si ses caractéristiques phonologiques ou morphologiques en permettent l'analyse, ou si le contexte et la situation sont suffisamment précis.

Quant à la restitution dans la langue d'arrivée de l'idée véhiculée par le terme technique, elle peut être compromise par :

- l'ignorance du terme correspondant dans la langue d'arrivée

- un "trou de mémoire " empêchant momentanément l'interprète d'évoquer dans la langue d'arrivée les mots nécessaires à l'expression de l'idée

- une rupture de l'équilibre d'interprétation

- des interférences linguistiques (l'incapacité momentanée de se détacher de la langue de départ)

- d'autres éléments perturbateurs (fatigue, bruits, etc.)

\section{LA PRÉPARATION}

Ces difficultés sont à l'origine de pertes d'information considérables, et compromettent gravement la mission de l'interprète, la restitution du message de l'orateur.

Le moyen de prévention universellement choisi est le travail de préparation, et le meilleur support envisageable pour celui-ci est constitué par l'ensemble des documents de la conférence (Seleskovitch $1968: 151$ ).

La documentation est lue, le vocabulaire repéré. La signification des mots est recherchée dans les documents mêmes, dans des dictionnaires, des ouvrages de référence, auprès des spécialistes.

La plupart des interprètes préparent de petits lexiques où les mots sont inscrits par ordre chronologique d'apparition, regroupés par affinités conceptuelles ou classés par ordre alphabétique. D'autres préfêrent annoter les documents mêmes, surtout les textes destinés à être lus.

La grande majorité des interprètes n'apprennent pas par cœur le vocabulaire ainsi constitué. Ils se contentent de le parcourir une ou deux fois avant la conférence, et comptent sur les opérations de lecture, de repérage, de marquage et de préparation du lexique pour fixer les mots dans leur mémoire. En cas de " panne », ils peuvent toujours consulter leur lexique en cabine (voir plus loin).

Une contrainte pratique fort fréquente est la brièveté des délais dont dispose l'interprète pour exploiter une documentation qui peut atteindre plusieurs centaines de pages. À l'origine, l'envoi tardif des documents par l'organisateur, mais aussi le caractère saisonnier de l'interprétation, susceptible de se traduire par la concentration de plus de dix conférences par mois pendant certaines périodes de l'année.

Dans ces conditions, la préparation ne peut être approfondie, et l'interprète est parfois obligé de faire une partie du travail en cabine, en étudiant de près un exposé qui lui a été attribué lors d'un partage des interventions entre les membres de l'équipe.

- La documentation est souvent très incomplète, et ne permet pas de cerner le vocabulaire effectif de la conférence. Il appartient alors à l'interprète de choisir une stratégie de préparation susceptible de lui être du plus grand secours.

L'auteur préconise une exploration "horizontale " avec approfondissements successifs : un survol du domaine permet de se repérer et de situer les thèmes destinés à être 
abordés durant la réunion ou susceptibles de l'être ; ces thèmes peuvent ensuite être étudiés de manière plus approfondie.

À ces fins, on consulte d'abord des encyclopédies, des ouvrages de vulgarisation, des livres scolaires, des spécialistes, puis des documents plus techniques, et de nouveau, des spécialistes.

Cette démarche permet à l'interprète d'acquérir un bagage cognitif utile face à des mots inconnus susceptibles d'apparaître en cours de conférence ; elle lui donne par ailleurs un aperçu du vocabulaire potentiel de la réunion et lui permet d'identifier ses caractéristiques (mots d'origine gréco-latine, mots anciens, mots composés, xénismes, etc.), lui facilitant ainsi l'analyse en situation.

\section{La recherche des "équivalents » en langue d'arrivée}

On a beaucoup souligné la nécessité de comprendre pour traduire (voir par exemple Seleskovitch $1975: 41-53$ ) ; le bien-fondé de la traduction par transcodage paraît en effet compromis par deux arguments importants :

- L'idée, devenue le bien commun de toute la linguistique actuelle, que chaque langue découpe dans le réel des aspects différents, et qu'elle découpe aussi le même réel en unités différentes (Mounin $1976: 48$ ). Un terme donné ne recouvre donc pas exactement la même réalité que son "équivalent " présumé dans une autre langue.

- L'idée de la polysémie obligatoire de la langue (voir Pergnier 1973 : 31-36). Celle-ci résulte mathématiquement du fait que dans les langues naturelles, " un nombre limité de signes a pour fonction de représenter une infinité de situations, une infinité d'éléments du monde extérieur, une infinité de représentations de la vérité par des locuteurs différents" (Guilbert 1975 : 15).

Un terme donné peut donc avoir plusieurs sens selon le contexte, et doit être traduit différemment selon le sens.

De cette formule : "comprendre pour traduire", certains tirent hâtivement la conclusion que seule une compétence certaine dans le domaine où se situe le texte permet de faire un travail de traduction sérieux (voir par exemple Kourganoff 1980 : 4).

Un certain niveau de compréhension est effectivement nécessaire à l'appréhension des liens logiques et fonctionnels entre les différents éléments de l'énoncé et à la sélection des termes appropriés en langue d'arrivée, mais il est généralement accessible aux traducteurs et interprètes professionnels (voir Gile 1980).

En ce qui concerne le travail de préparation, le problème de la polysémie est fortement atténué, voire totalement supprimé, par le contexte défini par la documentation. D'autre part, l'évolution parallèle des secteurs de pointe de la science et la technologie dans les différents pays depuis plusieurs dizaines d'années fait qu'en général, les termes techniques découpent la réalité d'une manière identique ou très proche.

Pour peu qu'ils disposent d'une documentation suffisante, les interprètes sont donc en mesure de trouver les équivalents contextuels appropriés des termes techniques posant des problèmes de traduction. Les cas réfractaires, peu nombreux en général, peuvent être résolus avec la collaboration des orateurs et des autres participants à la conférence.

Il convient de préciser qu'une différence importante distingue les besoins des interprètes de ceux des traducteurs. Ces derniers, dont le travail est conservé de manière permanente et doit parfois être publié, sont tenus de respecter des conditions de forme contraignantes : ils n'ont droit qu'à un vocabulaire "officialisé " dont sont exclus les xénismes, les sociolectes à diffusion trop limitée et les paraphrases. La situation des interprètes est toute autre. En effet, dans les conférences techniques, où la densité de l'information est considérable, les auditeurs se concentrent sur le fond et ne remarquent la 
forme que si les formules sont trop elliptiques, si les termes employés sont ignorés ou rares (voir Lederer $1978: 168$ ), ou s'ils les choquent pour une autre raison.

Les interprètes sont donc plus libres d'employer des néologismes, des xénismes, des barbarismes et des jargons très spécialisés, à condition que les termes soient clairs pour les auditeurs.

\section{LES TACTIQUES}

La prévention par la préparation est efficace quand la documentation est complète et le délai suffisant, plus incertaine quand ces deux conditions ne sont pas entièrement satisfaites, et plutôt aléatoire en l'absence de documents.

Par ailleurs, même dans des conditions optimales, l'interprète ne réagit pas toujours d'une manière spontanée et efficace.

Outre la stratégie de préparation, les interprètes ont donc recours à un ensemble de tactiques précises pour faire face aux difficultés terminologiques qui ne manquent pas d'apparaître en cours d'interprétation.

Le corpus montre que ces tactiques sont les mêmes, quelles que soient la formation, l'expérience et les langues de travail de l'interprète.

Lors de la première phase de l'interprétation, la phase d'écoute et d'analyse , les problèmes d'identification et de compréhension des termes techniques peuvent être combattus à l'aide de sept tactiques différentes :

1. L'interprète cherche à reconstituer le terme et son sens en s'aidant du contexte, de son bagage cognitif et des traits pertinents captés.

La réaction est naturelle en soi et fait partie du processus de réception ordinaire, déjà mentionné plus haut. La démarche devient " tactique " au sens du présent article quand l'effort est volontaire et mobilise l'attention de l'interprète. Elle est particulièrement fréquente quand l'accent étranger ou régional de l'orateur rend l'identification des mots difficile.

Quand elle aboutit, cette tactique permet la restitution intégrale du terme mal compris ou mal entendu, mais demande un certain temps, et surtout une grande énergie. Elle présente donc l'inconvénient d'un risque de rupture de l'équilibre d'interprétation, et de la perte à l'écoute de la suite immédiate du discours.

2. L'interprète demande l'aide de son collègue passif (qui est assis en cabine mais n'interprète pas). Celui-ci, qui peut consacrer toute son attention à l'écoute, entend et comprend souvent mieux que l'interprète actif. En outre, sa disponibilité lui permet de consulter un dictionnaire ou un autre document pour rechercher la solution.

Il suffit d'une hésitation, d'un coup d'œil, d'un geste, d'un mot écrit sur une feuille de papier avec un point d'interrogation pour que le collègue sache ce qui lui est demandé. Dans de bonnes équipes, il est d'ailleurs souvent possible d'anticiper les difficultés et de rechercher les solutions sans attendre.

Cette tactique est probablement la meilleure dont dispose l'interprète en cabine : peu coûteuse en temps et en énergie, elle lui donne de bonnes chances de retrouver l'information manquante.

Son utilisation suppose toutefois la présence d'un collègue passif en cabine, condition qui n'est pas toujours remplie :

- L'interprétation sollicite lourdement le système nerveux et appelle de fréquentes périodes de repos. Si la cabine est bilingue ou très active, un effectif de trois interprètes au moins est nécessaire pour qu'un interprète actif puisse se reposer pendant qu'un autre collègue prête main-forte à son successeur en cabine. Dans une équipe de deux, l'interprète passif ne peut rester en cabine en permanence, sous peine de voir son travail actif se dégrader rapidement par la suite. 
- Comme il a déjà été mentionné plus haut, les interprètes se trouvent parfois contraints d'achever leur préparation en séance.

- Enfin, très conscients de leurs faiblesses et de leurs défaillances, certains interprètes sont troublés par la présence de collègues qui les écoutent, et préferent travailler seuls.

3. En l'absence d'un collègue disponible à côté de lui, l'interprète peut rechercher une solution à son problème terminologique dans les documents dont il dispose en cabine : documents de travail, dictionnaires, listes de mots, etc.

Le travail de préparation a une grande influence sur l'efficacité de cette tactique : une bonne organisation des lexiques, un bon classement des documents, un bon repérage des termes techniques facilitent l'accès à l'information en séance.

Cette tactique peut être très intéressante quand un simple coup d'œil permet de trouver la solution au problème, sur un document de travail par exemple. La consultation d'un dictionnaire demande toutefois davantage de temps et d'effort, et n'est en général indiquée que pour une information importante et dans le cas d'un discours peu dense et peu rapide.

4. La simplification consiste à remplacer l'idée véhiculée, dont l'interprète a saisi la nature, mais non pas les contours précis, par une idée plus globale (push-pull amplifier est rendu par "l'amplificateur").

Cette tactique demande peu de temps et peu d'effort, mais implique l'abandon d'une partie de l'information. Cet abandon peut toutefois n'entraîner aucune perte pour l'auditeur, si le contexte ou un support visuel (diapositive, transparent, diagramme) sont suffisamment précis.

5. La reproduction approximative du son entendu par l'interprète est une tactique purement mécanique, dont l'efficacité est pourtant étonnamment bonne : les auditeurs rétablissent en effet souvent le mot et son sens grâce au contexte et à leur bagage cognitif, parfois sans même s'apercevoir des difficultés de l'interprète, surtout s'il s'agit de termes comportant des noms propres ("nombre de Froude", " antenne Yagi ») ou de termes phonologiquement proches de leurs équivalents contextuels en langue d'arrivée (voir à ce sujet Slama-Cazacu 1961).

6. Si l'orateur est dense et rapide, les interprètes ont besoin de toute leur énergie pour le suivre. Il arrive qu'une information mal perçue et jugée peu importante soit complètement omise en faveur de la suite.

Les omissions sont parfois des accidents plutôt que des tactiques, quand elles ne résultent pas d'un choix : il arrive qu'un effort excessif de mémoire ou de production du discours affaiblisse l'écoute, et l'interprète perd de l'information sans même s'en rendre compte.

Quand elle résulte d'une décision, l'omission implique l'abandon délibéré de la totalité de l'information véhiculée par le terme technique en question. La seule justification de cette démarche réside dans sa faible consommation de temps et d'énergie, qui permet à l'interprète de se concentrer sur une partie plus importante du discours et de sauvegarder l'essentiel.

Il convient de noter que dans la pratique, les conditions de travail sont telles que les omissions sont nombreuses, même chez des interprètes très compétents.

7. Quand l'interprète pense avoir perdu une information importante, il peut s'extraire momentanément de son personnage d'orateur et informer les auditeurs de la perte. Ceux-ci peuvent ne pas réagir, demander des précisions à l'orateur après son exposé, ou intervenir immédiatement.

Cette tactique n'est pas souvent employée : en effet, elle demande un certain temps et réduit la crédibilité de l'interprète, en dépit de l'honnêteté de la démarche. 
Toutes ces tactiques, à l'exception de la première, servent également lors du deuxième temps de l'interprétation, qui est la restitution du message. Pour surmonter une difficulté dans la réexpression d'une information véhiculée par un terme technique en langue de départ, les interprètes emploient également les tactiques suivantes :

8. L'explication ou la paraphrase : des termes n'existant pas dans la langue d'arrivée, inconnus de l'interprète ou momentanément insaisissables sont remplacés par des explications ou des paraphrases (action impulse est rendu par " recherche d'une ligne par sélecteur actionné par impulsions"). sidérable.

Cette tactique est efficace sur le plan informationnel, mais demande un temps con-

9. La "naturalisation": le terme technique en langue de départ est morphologiquement ou phonologiquement modifié pour être rapproché du vocabulaire de la langue d'arrivée (dans une interprétation dans le sens français-anglais, le mot " télédétection ", qui se traduit par remote sensing, a été rendu par teledetection, prononcé à l'anglaise).

Cette tactique d'" importation sauvage ", qui ne recueille pas les suffrages des défenseurs de la langue d'arrivée, n'est que rarement utilisée par les traducteurs. En interprétation, la primauté de la transmission informationnelle immédiate et l'évanescence de la parole justifient son emploi. En effet, faible consommatrice de temps et d'énergie, cette tactique est remarquablement efficace dans trois cas :

- Quand les langues de départ et d'arrivée sont très voisines (espagnol et français, par exemple), la transparence et l'étymologie commune et l'évocation de mots de la même famille permettent aux auditeurs de comprendre le sens de ces xénismes.

-Quand les " importations lexicales" sont monnaie courante entre la langue de départ et la langue d'arrivée : le japonais et l'hébreu, par exemple, ont adopté de très nombreux termes anglais, qui ccexistent d'ailleurs pacifiquement avec des synonymes autochtones. Dans le domaine technique, la naturalisation sauvage opérée par l'interprète débouche souvent sur des mots existant déjà dans le vocabulaire en usage, ou sur des créations qui leur sont proches.

- Quand les auditeurs ont une certaine habitude de la lecture de textes techniques dans la langue de départ sans pour autant la comprendre facilement à l'écoute. Ils identifient alors souvent sans peine le terme spécialisé prononcé par l'interprète comme ils le prononcent probablement eux-mêmes.

La prédominance de certaines langues dans les domaines scientifiques et techniques fait que ce cas est très fréquent.

10. La reproduction du mot en langue de départ, prononcé tel quel («le yellowfin ", prononcé à l'anglaise, a été employé dans une interprétation anglaisfrançais, le terme français « thon jaune » n'ayant pas été trouvé dans le feu de l'action).

L'efficacité de cette tactique est plus incertaine que celle de la "naturalisation ", car la prononciation étrangère est susceptible de rendre méconnaissable à l'oreille un terme connu ou compréhensible pour l'auditeur sous sa forme écrite. Indiquée entre l'italien et l'espagnol, par exemple, cette tactique est très risquée dans l'interprétation de l'anglais vers le japonais, où la "naturalisation » est par contre efficace.

11. Le transcodage (dans le domaine de la comptabilité nationale, maturity date, qui correspond à la "date d'échéance ", a été traduit par transcodage par "date de maturité ").

Cette tactique peut être efficace dans le champ lexical des technologies récentes, dont le vocabulaire progresse de manière parallèle dans les différents pays, mais la prudence s'impose quand les mots sont anciens : le transcodage en anglais de " tour d'échelle » (droit d'un propriétaire de passer des échelles sur la propriété de son voisin 
pour réparer ou entretenir le mur qui les sépare) aboutit à une formule peu claire pour l'auditeur anglophone.

\section{La sélection des tactiques}

Les différentes tactiques énumérées ne constituent pas des choix isolés, indépendants et définitifs. L'interprète use souvent de plusieurs démarches successives dans sa lutte contre la même difficulté. Il peut par exemple transcoder ou " naturaliser » tout en cherchant une solution dans son lexique, ou opter provisoirement pour l'omission en attendant la solution proposée par son collègue passif ; il peut aussi, s'il en a le temps, reproduire un terme tel quel, puis l'expliquer, ou omettre l'idée une première et une deuxième fois, puis recourir à une tactique dont le rendement informationnel est meilleur s'il voit que le terme réapparaît.

La sélection semble s'opérer essentiellement selon trois « lois ", dont l'importance relative est fortement influencée par des paramètres psychologiques :

(1) La recherche du meilleur rendement informationnel immédiat :

Pour D. Seleskovitch, l'interprétation est essentiellement " l'acquisition et la restitution de contenus cognitifs » (AICC 1979 : 34). Dans les conférences techniques, les interprètes perçoivent apparemment leur mission première comme consistant à transmettre de l'information, et tendent à choisir les tactiques les plus efficaces sur ce plan.

Cette loi favorise les tactiques assurant une restitution informationnelle intégrale (consultation d'un lexique où on est assuré de trouver le mot manquant) par rapport à celles dont la transmission informationnelle est incomplète ou aléatoire (simplification, "naturalisation").

\section{(2) La loi de la moindre interférence :}

Cette loi se focalise sur les incidences du choix de la tactique sur la restitution de la suite immédiate du discours original. Ces incidences sont fonction de l'énergie et du temps consommés par la tactique employée ; au regard de la loi de la moindre interférence, les omissions, par exemple, dont le rendement informationnel est nul, sont donc supérieures à l'explication, qui demande un certain temps et une certaine énergie, mais dont le rendement informationnel est élevé.

\section{(3) La loi du moindre effort :}

Cette loi est étrangère à toute considération technique, mais son effet est postulé dans une grande partie des activités humaines (Zipf 1965), et notamment dans le langage (Miller 1956). Elle apparaît en filigrane dans les travaux de K. Déjean-Le Féal (1978) et de B. Moser (1978) sur l'interprétation; I. Pinchuk (1977), pour sa part, l'érige en principe opératoire fondamental de la traduction.

La loi du moindre effort favorise à l'évidence, elle aussi, les tactiques les moins énergivores.

On notera que la seule tactique qui représente une solution optimale au regard de ces trois lois concurrentes est le recours au collègue passif; d'où l'intérêt des équipes de trois ou quatre interprètes.

L'interprète obéit plus ou moins consciemment à ces trois lois ; l'importance relative de chacune varie en fonction de facteurs psychologiques et déontologiques dont les principaux méritent qu'on s'y arrête.

La conscience professionnelle privilégie les deux premières lois, qui visent un rendement informationnel optimum, par rapport à la troisième. 
La fatigue, de mauvaises conditions de travail, l'absence de documents, un désintérêt apparent des organisateurs et des délégués à l'égard de l'interprétation sont susceptibles d'inverser les priorités et de favoriser la loi du moindre effort.

La recherche du maintien de la crédibilité est un autre élément important qui entre en jeu. L'interprète honnête devrait en principe notifier ses auditeurs des pertes d'information. Toutefois, une telle démarche, si elle se renouvelle plusieurs fois au cours d'une séance, compromet gravement sa crédibilité, et affaiblit par là l'impact du message de l'orateur. C'est pourquoi la plupart des interprètes y renoncent, quitte à expliquer aux organisateurs et aux délégués que des pertes informationnelles importantes sont inévitables quand les conditions de travail sont mauvaises.

\section{LES PARAMÈTRES DE LA QUALITÉ}

La part des termes techniques dont la charge informative est partiellement ou totalement perdue en interprétation simultanée est très variable. Dans le corpus, elle atteint communément plus de $80 \%$ d'un côté, et moins de $10 \%$ de l'autre ; une présentation globale de ces statistiques aurait un intérêt plus anecdotique qu'instructif.

Toutefois, l'analyse permet de dégager trois paramètres qui semblent avoir une influence déterminante sur la capacité de réaction de l'interprète face aux difficultés terminologiques :

\section{(1) La vitesse de l'orateur}

La vitesse de l'orateur est de loin le facteur le plus important. S'il est difficile d'isoler et de déterminer quantitativement son influence, il est néanmoins clair que plus le débit de l'orateur augmente, plus grande est la proportion d'informations perdues, et qu'à partir d'un certain seuil, aucun autre paramètre ne peut compenser cet effet.

\section{(2) Le mode d'énonciation du discours : lecture ou improvisation}

Les discours lus sont réputés plus difficiles à interpréter en simultanée que les interventions spontanées, et ce en raison de leur densité, leur rythme, et l'absence d'idéation chez le lecteur (K. Déjean-Le Féal 1978).

Par contre, seuls les discours lus donnent à l'interprète la possibilité de prendre connaissance de tous les termes techniques à l'avance. L'interprétation des textes lus débouche souvent sur des énoncés peu clairs et entâchés d'interférences linguistiques qui ne facilitent pas la compréhension (K. Déjean-Le Féal 1981 : 95), mais le corpus lui reconnaît une nette supériorité dans la restitution des informations véhiculées par les termes spécialisés.

\section{(3) L'expérience et les connaissances spécialisées des interprètes}

Nombreux sont les interprètes qui affirment ne retenir les termes techniques que le temps de la conférence, pour commencer à les oublier sitôt après (AICC $1979: 64$ ). D'autres pensent acquérir un vocabulaire technique de plus en plus vaste au fil des années (Lederer $1973: 118$ ).

Le corpus semble corroborer la deuxième opinion : statistiquement parlant, la proportion des termes techniques connus des interprètes chevronnés et inconnus de leurs cadets est telle qu'elle permet souvent aux uns de faire un discours cohérent là ou les autres baissent les bras.

Le bagage cognitif de l'interprète ayant une formation ou une expérience professionnelle dans le domaine dont traite la conférence constitue lui aussi un atout considérable. Cependant, son utilité décroît rapidement à mesure que l'on s'éloigne de la spontanéité de l'interprète : un ancien professionnel des statistiques devenu interprète comprend mieux les raisonnements statistiques que ses collègues non initiés, mais n'est 
pas beaucoup mieux armé qu'eux sur le plan terminologique dès que l'on passe aux statistiques démographiques.

D'autres paramètres sont faciles à énumérer, bien que leur influence soit plus difficile à cerner. Il en est deux, en particulier, qui méritent d'être mentionnés, car le corpus fait apparaître à leur propos des phénomènes qui ne concordent pas avec les idées et méthodes qui ont cours dans certains milieux importants de la profession :

\section{(4) La stratégie de préparation choisie}

D'une manière générale, la préparation cognitive semble nettement moins efficace que la préparation terminologique : l'image de l'interprète honnête homme qui s'efforce de comprendre par l'exégèse du discours, et qui restitue ensuite des idées parfaitement assimilées doit s'effacer, dans le cas du discours technique, en faveur de celle du technicien qui s'occupe en priorité des détails.

Certes, le survol d'un domaine par le biais d'un ouvrage de vulgarisation (ou par une autre méthode) permet, dans une négociation, dans une réunion politique, de comprendre les intérêts en jeu, les objectifs, les grandes orientations, et d'éviter maints pièges. Ce type de préparation est néanmoins moins efficace dans les conférences techniques, où les problèmes sont circonscrits dans de petits segments d'énoncé. Pour citer $\mathbf{M}$. Lederer (1981:53):

Ce qui différenciera toujours la situation de l'interprète de celle d'auditeurs normaux, c'est que le sens général des discours le concerne moins que leurs molécules, les unités de sens.

Pour bien suivre l'orateur, l'interprète doit comprendre le discours au niveau atomique, moléculaire et intermoléculaire ; il doit saisir les relations fonctionnelles à l'intérieur des phrases et segments de phrases, ainsi que les enchaînements. Au-delà, les rendements des efforts d'approfondissement décroissent rapidement.

Le corpus présente de nombreux exemples d'interprètes ayant bien interprété un exposé sans le comprendre dans son ensemble. Dans l'idéal, l'élucidation des principes fondamentaux des sciences et techniques doit précéder la recherche terminologique (Seleskovitch 1981 : 44); dans la pratique, un travail de bonne qualité est possible sans cette étape préalable.

\section{(5) La langue d'expression de l'interprète}

Il est généralement admis à l'AIIC que l'interprète doit travailler vers sa langue " $\mathbf{A}$ " (langue maternelle ou assimilée). L'interprétation vers la langue " $\mathbf{B}$ " (autre langue active) est considérée comme un mal nécessaire imposé par les réalités du marché (Skuncke 1976 : 104).

Pour D. Seleskovitch, "à qualité égale d'interprète, l'interprétation en «A » est toujours supérieure à l'interprétation en «B»»(1968:224). E. Bros-Brann va plus loin et affirme que la véritable interprétation, qui est autre chose que le simple transcodage de l'original, ne peut se faire que vers la langue " $\mathbf{A}$ " (1976:17).

Le corpus ne fait pas ressortir une telle différence d'une manière très nette. Il est vrai que les maladresses de style et fautes de langue sont souvent plus nombreuses chez les interprètes travaillant de " $\mathbf{A}$ " en " $\mathbf{B}$ ", mais les termes techniques mal compris et mal traduits ou omis sont parfois plus nombreux chez les interprètes travaillant de $B$ en « $\mathbf{A}$ ». Il semblerait en effet que la meilleure qualité des structures de réception en " $\mathbf{A}$ " compense en partie ou entièrement la capacité de production inférieure en $" \mathbf{B}$ ". 


\section{CONCLUSION}

Le recueil et l'exploitation des données sur l'interprétation dans les conférences techniques ne font que commencer, et des analyses statistiques fines n'ont pu être réalisées jusqu'à présent. Nous pensons d'ailleurs qu'à ce stade assez peu avancé de l'exploration de l'interprétation simultanée, les grandes lignes de convergence n'ont pas encore été suffisamment dégagées et exploitées pour que la recherche se tourne vers des phénomènes plus fins et à caractère plus aléatoire.

En matière terminologique, les éléments réunis dans le corpus semblent confirmer que dans les interventions très techniques, la majeure partie des pertes informationnelles de l'interprète sont attribuables à son incapacité de saisir ou de restituer les informations véhiculées par les termes spécialisés.

Étant donné le nombre et la variété des mots susceptibles d'être employés à chaque conférence, l'interprète ne peut travailler de manière efficace sans une préparation spécifique avant chaque réunion. Le corpus semble indiquer que le meilleur rendement informationnel est obtenu par un travail essentiellement terminologique sur des textes destinés à être lus ou paraphrasés.

La recherche d'une restitution aussi complète que possible de l'information conduit apparemment, dans les conférences techniques, à des préférences et à des méthodes peu orthodoxes par rapport aux idées généralement admises :

- Le travail le plus efficace est très proche de la traduction à vue, et diffère sensiblement de l'interprétation d'un discours improvisé.

- La supériorité présumée du travail du " $B$ » vers le " $A$ » est remise en question. Cette observation appelle d'ailleurs des précisions sur le plan linguistique, que de futurs travaux devraient pouvoir fournir. L'identification des faiblesses des langues «B " par rapport aux langues " $\mathbf{A}$ » en interprétation devrait ouvrir la voie à des efforts de perfectionnement linguistique spécialisé qui ne figurent pas aux programmes des écoles à l'heure actuelle.

- Dans le travail de préparation, l'importance de la recherche terminologique est déterminante. Une prise de conscience dans ce domaine devrait pouvoir s'accompagner d'une certaine réorganisation des méthodes. Les interprètes, dont les besoins terminologiques sont quantitativement supérieurs à ceux des traducteurs techniques, sont en effet très mal équipés en dictionnaires et en lexiques. Il en résulte parfois un travail approximatif que de bons outils terminologiques permettraient d'éviter.

Mais la préparation et les tactiques employées en situation ne peuvent résoudre l'ensemble des problèmes terminologiques, qui occasionnent souvent des pertes d'information considérables (il s'agit des pertes au niveau de l'interprète ; la question de leurs incidences sur le taux de réception des auditeurs n'ayant pas encore été étudiée, nous partons de l'hypothèse de la proportionnalité).

En fait on pourrait parler de deux types d'interprétation : dans la première, du type classique, l'interprète se substitue véritablement à l'orateur et transmet sa personnalité et son style en même temps que l'information ; dans la seconde, l'interprétation technique, il est un intervenant distinct de l'orateur, qui s'efforce de restituer aussi complètement que possible une information essentiellement impersonnelle. Les qualités et aptitudes requises pour ces deux "spécialités " divergent sensiblement et semblent appeler des formations et des méthodes spécifiques.

\section{BIBLIOGRAPHIE}

AICC (1979) : Enseignement de l'interprétation, dix ans de colloques (1969-1979), Genève.

BROS-BRAN, Éliane (1976) : Critical Comments on H.C. Barik's article "Interpreters talk a lot, among other things ", Bulletin de l'AICC IV/1, Genève, mars. 
DÉJEAN-LE FÉAL, Karla (1978) : Lectures et improvisations — incidences de la forme de l'énonciation sur la traduction simultanée, doctorat de $3^{\mathrm{e}}$ cycle, Université de la Sorbonne nouvelle, Paris.

DÉJEAN-LE FÉAL, Karla (1981) : "L'enseignement des méthodes d'interprétation ", dans Jean Delisle, l'Enseignement de l'interprétation et de la traduction de la théorie à la pédagogie, Ottawa, Éditions de l'Université d'Ottawa.

GILE, Daniel (1980) : "Textes spécialisés : techniciens ou traducteurs? ", dans Traduire, no 105, décembre, IV.

GILE, Daniel : «Des difficultés de la transmission informationnelle en interprétation simultanée ", article à paraître.

GUILBERT, Louis (1975) : la Créativité lexicale, Paris, Larousse.

HÖRMANN, Hans (1972) : Introduction à la psycholinguistique, Paris, Larousse.

KOURGANOFF, Vladimir (1980) : "Quelques traquenards du thème scientifique anglais ", dans Traduire, $\mathrm{n}^{\circ} 103, \mathrm{juin} / \mathrm{I}$.

LEDERER, Marianne (1973) : "L'approche de l'inconnu ", dans E. Weintraub, M. Lederer, J. de Clarens : "Enseigner l'interprétation ", Études de linguistique appliquée, n $^{\circ} 12$, octobre-décembre.

LEDERER, Marianne (1978) : la Traduction simultanée - fondements théoriques, Paris, Université ParisSorbonne.

LEVELT, W.J.M. (1978) : " A Survey of Studies in Sentence Perception : 1970-1976", dans W.J.M. Levelt et G.B. Flores d'Arcais, Studies in the Perception of Language, John Wiley and Sons.

LEVINSON, S. et M. LIBERMAN (1981) : "La reconnaissance de la parole par ordinateur ", dans Pour la science, édition française de Scientific American, $n^{\circ} 44$, juin.

LOCHNER, R.K. (1976) : "Conference Interpretation and the Modern World", dans BABEL, 22/3.

MILLER, George A. (1956) : Langage et communication, Paris, PUF.

MOSER, Barbara (1977) : "A Hypothetical Model and its Practical Applications ", dans D. Gerver and H.W. Sinaiko : Language Interpretation and Communication, New York and London, Plenum Press. MOUNIN, Georges (1976) : les Problèmes théoriques de la traduction, Paris, Gallimard.

PERGNIER, Maurice (1973) : "Traduction et théorie linguistique ", dans Études de linguistique appliquée, no 12 , octobre-décembre.

PINCHUK, Isadora (1977) : Scientific and Technical Translation, London, André Deutsch.

SELESKOVITCH, Danica (1968) : l'Interprète dans les conférences internationales, Paris, Minard.

SELESKOVITCH, Danica (1975) : Langages, langue et mémoire, Paris, Minard.

SKUNCKE, Marie-France (1976) : "Rapport du colloque sur l'enseignement de la simultanée », Bulletin de I'AICC, IV $/ 2$, août.

SLAMA-CAZACU, Tatiana (1961) : Langage et contexte, La Haye, Mouton.

ZIPF, G.K. (1965) : Human Behavior and the Principle of Least Effort, New York, Hefner Publishing Co. 